\title{
В. В. ГРЕБЕНИКОВ, Р.В. ГАМАЛЕЯ
}

\section{ИССЛЕДОВАНИЕ ВЛИЯНИЯ КОНФИГУРАЦИИ МАГНИТНОЙ СИСТЕМЫ НА МОМЕНТНЫЕ ХАРАКТЕРИСТИКИ МАГНИТНОГО РЕДУКТОРА}

Наведено результати дослідження характеристик електродвигуна і редуктора 3 неодимовими магнітами для мотор-колеса електромобіля. Для заданих габаритів мотор-колеса з магнітним редуктором (зовнішній діаметр, осьова довжина електродвигуна і редуктора) визначено конфігурацію і розміри магнітної системи електродвигуна і редуктора, при якій досягається необхідне значення моменту в заданому діапазоні частоти обертання з урахуванням їздових циклів. Розрахунок характеристик досліджуваного електродвигуна і редуктора виконаний в програмному пакеті Infolytica Magnet.

Ключові слова: мотор-колесо, електродвигун і редуктор на постійних магнітах, електромагнітний момент, робочі характеристики, чисельне моделювання, європейський їздовий цикл.

Приведены результаты исследования характеристик электродвигателя и редуктора с неодимовыми магнитами для мотор-колеса электромобиля. Для заданных габаритов мотор-колеса с магнитным редуктором (внешний диаметр, осевая длина электродвигателя и редуктора) определена конфигурацию и размеры магнитной системы электродвигателя и редуктора, при которой достигается требуемое значение момента в заданном диапазоне частоты вращения с учетом ездовых циклов. Расчет характеристик исследуемого электродвигателя и редуктора выполнен в программном пакете Infolytica Magnet.

Ключевые слова: мотор-колесо, электродвигатель и редуктор на постоянных магнитах, электромагнитный момент, рабочие характеристики, численное моделирование, европейский ездовой цикл.

Magnetic gears based on permanent magnets become very popular and promising because they are able to transmit torque without mechanical contact, do not create additional noise, do not require lubrication, have high efficiency and reliability, are more durable, which makes it possible to use them instead of mechanical gearboxes, including for motor-wheels of electric vehicles. Due to the design features of the magnetic gearbox, during its operation, pulsations of the transmitted torque occur. The main cause of pulsations is the presence of a cogging torque. When designing a magnetic gearbox, one should strive to ensure that the amount of cogging torque is minimal, since it causes vibrations and noise in the equipment and leads to a deterioration in the performance of the magnetic gearbox, and therefore decreases the efficiency of the motor-wheel. As a result of numerical studies, it was found that with an integer value of the reduction coefficient, the cogging torque is several times larger than for a non-integer value. For the given dimensions of the gearbox (outer diameter, axial length), the configuration and dimensions of the magnetic gearbox system are determined, at which the specified values of the gearbox moments are achieved at the minimum values of the cogging torque. It seems highly technological to produce a magnetic gear modulator consisting of fixed steel segments as a single unit, steel segments in which are connected by arches and the modulator can be made by stamping or laser cutting electrical steel sheets. As a result of numerical studies, the optimum thickness of the connecting arches and their location between the steel elements of the modulator are determined, at which the specified value of the gearbox torque and the technological effectiveness of its manufacture and assembly are provided. The calculation of the characteristics of the researching magnetic gear was made in the software package Infolytica Magnet.

Keywords: motor-wheel, magnetic gearbox with permanent magnets, electromagnetic moment, jagged moment, numerical simulation

Введение. Магнитные редукторы на основе постоянных магнитов становятся весьма востребованными и перспективными, поскольку имеют ряд преимуществ по сравнению с механическими. Магнитные редукторы способны передавать крутящий момент без механического контакта, не создают дополнительного шума, не требуют смазки, имеют высокую эффективность и надежность, более долговечны, что дает возможность применять их вместо механических редукторов. В настоящее время магнитные редукторы используются для различных применений, в том числе для мотор-колес электромобилей $[1,2]$.

Для электромобиля в соответствии с ездовым циклом $[2,13]$, максимальная частота вращения колеса составляет 1000 об/мин, использование электродвигателя без редуктора при такой частоте вращения нецелесообразно, поскольку габариты электродвигателя будут весьма существенными. Поэтому в последнее время интенсифицируются исследования мотор-колес с электродвигателем и магнитным редуктором на основе редкоземельных постоянных магнитах [2-6], хотя есть исследования магнитных редукторов и на основе ферритовых постоянных магнитов [7]. Как правило, для мотор-колес используются цилиндрические магнитные редукто- ры, однако весьма перспективным является применение циклоидальных магнитных редукторов, которые характеризуется предельной плотностью крутящего момента и очень высоким передаточным числом [8]. В статье [9] продемонстрировано, что магнитные редукторы, интегрированные с электродвигателем на постоянных магнитах даже предпочтительнее механических редукторов из-за их сравнительно более высокого удельного крутящего момента $\left(\kappa \mathrm{H} \cdot \mathrm{m} / \mathrm{M}^{3}\right)$.

Однако магнитные редукторы не лишены своих недостатков, при определенном соотношении числа пар полюсов значение зубцового момента (в англоязычной литературе-cogging torque) может быть весьма значительным, а значит, коэффициент полезного действия магнитного редуктора будет не высоким. Если это явление не учтено, это может привести к переоценке удельного крутящего момента.

Анализ состояния вопроса. Магнитный редуктор цилиндрического типа, состоит из внутреннего высокоскоростного ротора, модулятора из неподвижных стальных элементов и внешнего низкоскоростного ротора. В силу особенностей конструкции магнитного редуктора, во время его работы возникают пульсации передаваемого крутящего момента. Основной причиной пульсаций является наличие

(C) В. В. Гребеников, Р.В. Гамалея, 2019 
зубцового момента. Таким образом, при проектировании магнитного редуктора следует стремиться, чтобы величина зубцового момента была минимальной, поскольку она вызывает вибрации и шумы в оборудовании и приводит к ухудшению рабочих характеристик магнитного редуктора, а значит и к уменьшению коэффициента полезного действия мотор-колеса $[1,10]$.

Модулятор магнитного редуктора, состоящий из не подвижных стальных сердечников, должен быть выполнен из шихтованной электротехнической стали. Однако, если их изготавливать как отдельные элементы, то конструкция модулятора получается не технологичной при изготовлении и сборке. В работе [11] исследуются влияние на характеристики магнитного редуктора различных конструкций модулятора, и показано, что за счет изменения дизайна модулятора может быть достигнут компромисс между моментными характеристиками и технологичностью изготовления.

Следует отметить, что исследованию характеристик магнитного редуктора применительно к мотор-колесу электротранспорта не уделено достаточно внимания. В частности, представляет интерес исследование влияния числа пар полюсов высокоскоростного и низкоскоростного ротора на величину зубцового момента и моментов высокоскоростного и низкоскоростного ротора, а также влияние на моментные характеристики редуктора конфигурации неподвижных стальных сегментов модулятора.

Цель исследований. Для заданных габаритов магнитного редуктора (внешний диаметр, осевая длина редуктора) определить конфигурацию и размеры магнитной системы, при которой достигаются заданные значения моментов редуктора и минимальные значениях зубцового момента, и обеспечивается технологичность изготовления и сборки.

Постановка задачи. При исследовании характеристик магнитного редуктора, применяемого для мотор-колеса электромобиля среднего класса, следует учитывать, что габариты определяются пространством, которое задается размерами автомобильного диска R16. Чтобы мотор-колесо с редуктором разместилось в пространстве этого диска, размеры магнитного редуктора должны быть не более: диаметр 390 мм, аксиальная длина - 50 мм.

На рис. 1 показан общий вид магнитного редуктора, который имеет внутренний высокоскоростной (BC) ротор, состоящий из стального замыкателя 1 и постоянных магнитов 2, внешний низкоскоростной (HC) ротор, состоящий из постоянных магнитов 4 и стального замыкателя 5, а также модулятора, состоящего из стальных неподвижных сегментов 3.

Передаточное отношение магнитного редуктора и число неподвижных стальных сегментов определяется соотношением между числом пар полюсов на низкоскоростном (внешнем) и высокоскоростном (внутреннем) роторе:

$$
G_{\text {мр }}=p_{\text {вс }} / p_{\text {нс }} ; \omega_{\text {вс }}=-G_{\mathrm{r}} \omega_{\text {нс }} ; n_{\mathrm{s}}=p_{\text {нс }}+p_{\text {вс }},
$$

где $G_{\text {мр }}$ - передаточное отношение магнитного редуктора; $p_{\text {нс }}$ - число пар полюсов низкоскоростного ротора; $p_{\text {вс }}$ число пар полюсов высокоскоростного ротора; $n_{\mathrm{c}}$ - число неподвижных стальных сегментов; $\omega_{\text {нс }}$ - частота вращения низкоскоростного ротора; $\omega_{\text {вс }}$ - частота вращения высокоскоростного ротоpa (низкоскоростной и высокоскоростной ротор вращаются в противоположных направлениях).

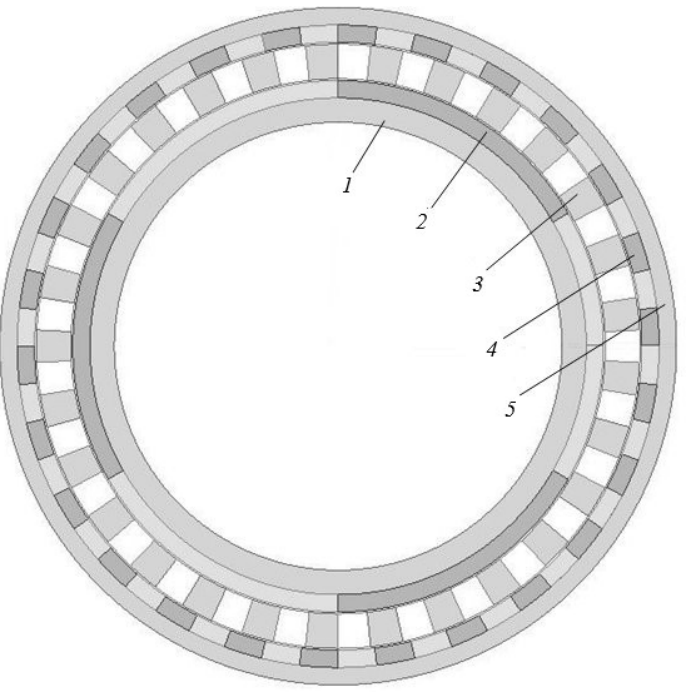

Рис. 1 - Общий вид магнитного редуктора

Конфигурация магнитной системы магнитного редуктора существенным образом влияет на их характеристики [7-10]. Максимальная частота вращения мотор-колеса для загородного ездового цикла составляет $\omega_{\text {нс }}=1000$ об/мин $[12,13]$. Ясно, что при заданных габаритах магнитного редуктора масса постоянных магнитов не зависит от передаточного отношения редуктора, а чем выше передаточное отношение магнитного редуктора, тем выше может быть частота вращения внутреннего ротора, т.е. частота вращения ротора электродвигателя и, соответственно, меньше габариты электродвигателя.

Поэтому при коэффициенте редукции равном, например, $G_{\text {мр }}=10$, максимальная частота вращения ротора электродвигателя должна быть $\omega_{\text {вс }}=10000$ об/мин, а при $G_{\text {мр }}=30-$ соответственно $\omega_{\mathrm{Bc}}=30000$ об/мин. Однако для магнитного редуктора с большим передаточным числом требуется большое количество неподвижных стальных сегментов с относительно небольшой толщиной, которые могут частично насыщаться, а также может быть чрезмерная утечка потоков между этими неподвижными сегментами. В связи с этим передаточное отношение магнитного редуктора применительно к мотор-колесам транспортных средств, как правило, применяется в диапазоне $G_{\text {мp }}=8-10$ [2-5].

Параметры исследуемого редуктора: толщина постоянных магнитов, высота неподвижных стальных сегментов, варьировались таким образом, чтобы обеспечить величину электромагнитного момента на внешнем низкоскоростном роторе не менее $M_{(\mathrm{HC}) \max }=700 \mathrm{H} \cdot$ м. При этом задавались следующие соотношения числа пар полюсов редуктора $\left(p_{\mathrm{вc}} / p_{\text {нс }}\right)$ : 
(3 / 24); (3/25); (3 / 26); (3 / 27); (3 / 28). Величина зазора между внешним ротором и стальными сегментами $-\delta_{1}=1$ мм, а также между внутренним ротором и стальными сегментами $-\delta_{2}=1$ мм.

Результаты численного исследования. Важнейшими характеристиками магнитного редуктора как устройства, передающего и преобразовывающего механическую энергию вращения, являются зависимости моментов, действующих на его роторы как функции положения (углов поворота роторов). Это периодические функции, максимумы и минимумы которых при одинаковых геометрических размерах и используемых материалах зависят от соотношения количеств пар полюсов на роторах магнитного редуктора.

Перед проведением численных исследований характеристик магнитного редуктора были проведены в пакете Infolytica Magnet расчеты магнитного редуктора из [6], конфигурация магнитной системы которого аналогична рис. 1. В статье [6] приводятся численные и экспериментальные значения параметров исследуемого магнитного редуктора. Основные размеры этого редуктора следующие: число пар полюсов низкоскоростного ротора $-p_{\text {нс }}=22$; число пар полюсов высокоскоростного ротора $-p_{\text {вс }}=4$; внешний диаметр низкоскоростного ротора - 130 мм; внутренний диаметр низкоскоростного ротора 110 мм; внешний диаметр высокоскоростного ротора - 76 мм; толщина магнитов - 5 мм; рабочие зазоры - 1 мм; аксиальная длина редуктора - 15 мм.

В результате численного моделирования получены следующие результаты: максимальный момент на высокоскоростном роторе $-10,81 \mathrm{H} \cdot \mathrm{M}$, в [6] $10.58 \mathrm{H} \cdot$ м, т.е. расхождение - $2,1 \%$; максимальный момент на низкоскоростном роторе $-1,98 \mathrm{H} \cdot$ м, в [6] $-1,95 \mathrm{H} \cdot$ м, т.е. расхождение $-1,5 \%$. Таким образом, разработанная в пакете Infolytica Magnet модель магнитного редуктора адекватна физической модели из статьи [6].

На первом этапе исследовалось влияние соотношения числа пар полюсов (коэффициента редукции) на максимальные значения зубцового момента и моментов, действующих на роторы магнитного редуктора.

На рис. 2 приведены максимальные значения моментов на высокоскоростном $\left(M_{(\text {вс)max }}\right)$, низкоскоростном $\left(M_{(\text {нс) } \max }\right)$ роторе и зубцового момента $M_{3}$ для различных соотношений пар полюсов. Зубцовый момент магнитного редуктора, действующий на высокоскоростной ротор в заданном положении определялся, как момент $M_{3}$ необходимый для удержания ротора в этом положении в то время, когда низкоскоростной ротор находится в положении устойчивого магнитного равновесия $\left(M_{\mathrm{Hc}}=0, d M_{\mathrm{HC}} / d \alpha_{\mathrm{Hc}}<0\right.$, где $\alpha_{\text {нс }}$ - угол поворота низкоскоростного ротора). Таким образом, зубцовый момент также является периодической функцией угла поворота ротора (рис. 3) с характерными максимумами и минимумами.

Следует отметить, что зубцовый момент существенно зависит от коэффициента редукции т.е. от- ношения числа пар полюсов низкоскоростного ротора к числу пар полюсов высокоскоростного ротора (рис. 2, 3). В табл. 1 приведены максимальные значения зубцового момента $M_{3}$ на высокоскоростном роторе и его составляющие для магнитных редукторов с коэффициентами редукции $G_{\mathrm{mp}}=8$, соотношение пар полюсов - $(3 / 24), G_{\mathrm{mp}}=8.67-(3 / 26)$, $G_{\text {мр }}=9-(3 / 27)$ и $G_{\text {мр }}=9.33-(3 / 28)$. Составляющие зубцового момента - это моменты, действующие на отдельные полюса ротора $M_{3(\mathrm{n} 1)}, M_{3(\mathrm{n} 2)}, M_{3(\mathrm{n} 3),} M_{3(\mathrm{~s} 1) \text {, }}$ $M_{3(\mathrm{~s} 2),} M_{3(\mathrm{~s} 3)}$ и момент, действующий на стальной замыкатель ротора $M_{3 \text { (ring). }}$

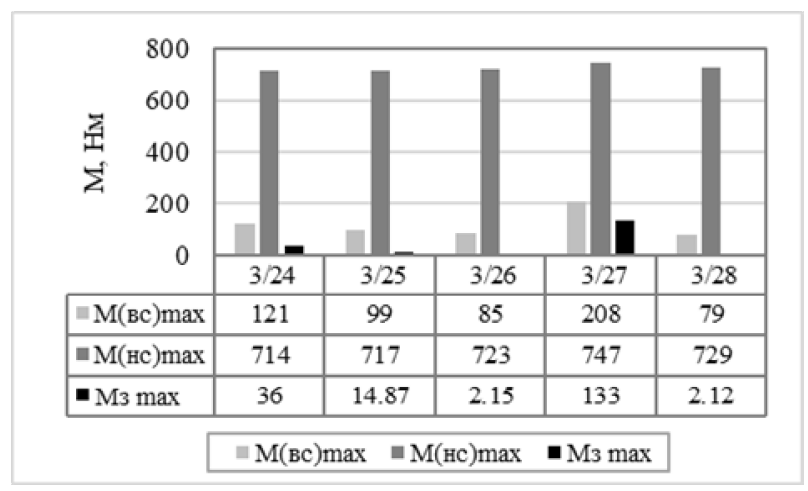

Рис. 2 - Зависимость максимальных значений момента на высокоскоростном $\left(M_{(\text {вс) max }}\right)$, низкоскоростном $\left(M_{(\text {нс)max }}\right)$ роторе и зубцового момента от соотношения пар полюсов.

Для целочисленных значений коэффициента редукции зубцовый момент существенно выше, так как в этом случае каждый из полюсов ротора находится в геометрически одинаковом положении относительно неподвижных ферромагнитных элементов модулятора и моменты, действующие на эти полюса, имеют одинаковые знаки и суммируются. Для нецелочисленных значений коэффициента редукции моменты, действующие на полюса в силу их различного положения относительно ферромагнитных элементов модулятора, разнонаправлены и результирующий момент оказывается меньше.

Таблица 1 - Составляющие зубцового момента на высокоскоростном роторе магнитного редуктора

\begin{tabular}{|l|r|r|r|r|r|}
\hline & $(3 / 24)$ & $(3 / 25)$ & \multicolumn{1}{|c|}{$(3 / 26)$} & \multicolumn{1}{c|}{$(3 / 27)$} & $(3 / 28)$ \\
\hline$M_{3}$ & 36,14 & $-14,87$ & 2,15 & $-132,65$ & 2,13 \\
\hline$M_{3(\mathrm{n} 1)}$ & 5,48 & $-5,55$ & 15,42 & $-23,00$ & 14,93 \\
\hline$M_{3(\mathrm{n} 2)}$ & 5,48 & $-8,09$ & 4,92 & $-23,36$ & $-16,35$ \\
\hline$M_{3(\mathrm{n} 3)}$ & 5,48 & 9,22 & $-14,99$ & $-23,25$ & 1,04 \\
\hline$M_{3(\mathrm{~s} 1)}$ & 6,06 & 9,22 & 17,6 & $-22,99$ & $-3,32$ \\
\hline$M_{3(\mathrm{~s} 2)}$ & 6,06 & $-5,56$ & $-6,17$ & $-23,24$ & $-9,16$ \\
\hline$M_{3(\mathrm{~s} 3)}$ & 6,06 & $-8,09$ & $-5,20$ & $-22,9$ & 12,89 \\
\hline$M_{3(\text { ring })}$ & 1,52 & $-6,02$ & $-9,43$ & 6,09 & 2,10 \\
\hline
\end{tabular}

Из данных приведенных на рис. 2 и табл. 1 следует, что варианты соотношения полюсов $(3 / 26)$ и (3/28) более предпочтительны, так как, обладая примерно одинаковыми возможностями по передаче механической энергии, имеют самые низкие значе- 
ния зубцовых моментов и как следствие более высокий коэффициент полезного действия редуктора.

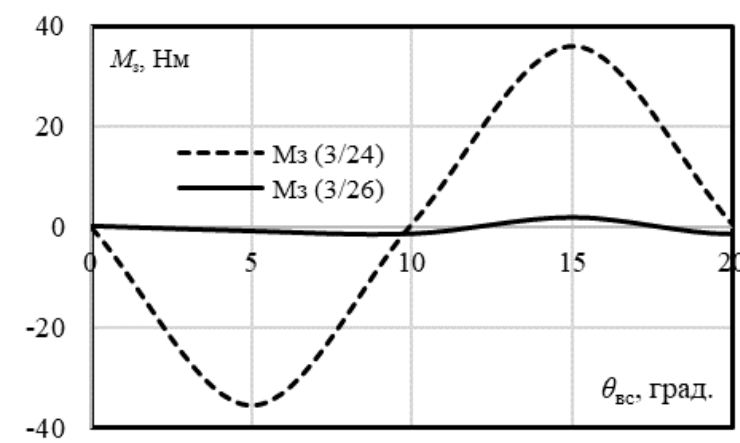

Рис. 3 - Зависимость зубцового момента от угла поворота низкоскоростного ротора для соотношения пар полюсов $(3 / 24)$ и $(3 / 26)$.

Таким образом, в дальнейших исследованиях рассматривался магнитный редуктор с коэффициентом редукции $G_{\text {мр }}=8.33$ и соотношением пар полюсов $\left(p_{\text {вс }} p_{\text {нс }}\right)-(3 / 26)$. Для этого соотношения на рис. 4 приведены зависимости моментов на высокоскоростном и низкоскоростном роторах от угла поворота низкоскоростного ротора. Для любого угла отношения этих моментов близко к коэффициенту редукции (не равно в виду наличия зубцового момента).

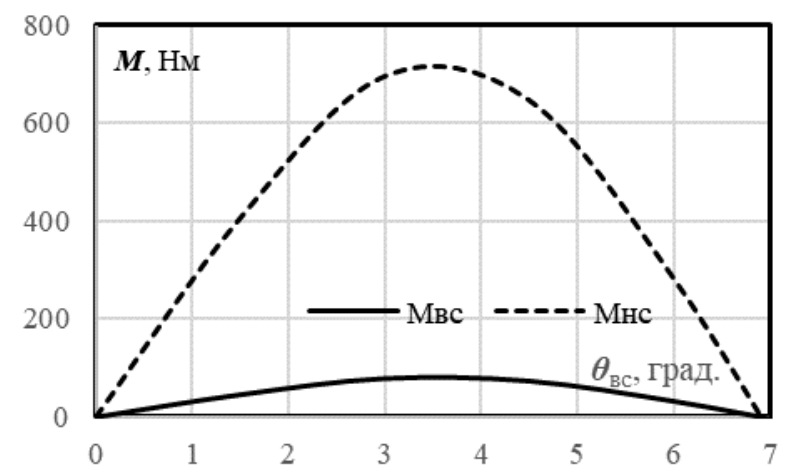

Рис. 4 - Зависимость момента на высокоскоростном и низкоскоростном роторах от угла поворота низкоскоростного ротора для соотношения пар полюсов $(3 / 26)$.

На рис. 5 представлена общая картина распределения магнитного поля редуктора, полученная в результате численного моделирования методом конечных элементов при помощи программного пакета Infolytica Magnet. При этом учитывались нелинейные магнитные характеристики как электротехнической стали, так и постоянных магнитов. Результаты моделирования показали, что среднее значение магнитной индукции в ферромагнитных элементах роторов магнитного редуктора не превышает 1,6 Тл, модулятора - 1,7 Тл, существенных потоков рассеивания не наблюдается.

Таким образом, заданные геометрические параметры редуктора позволяют концентрировать магнитное поле в стальных элементах модулятора и обеспечивают эффективную передачу крутящего момента от одного ротора к другому.

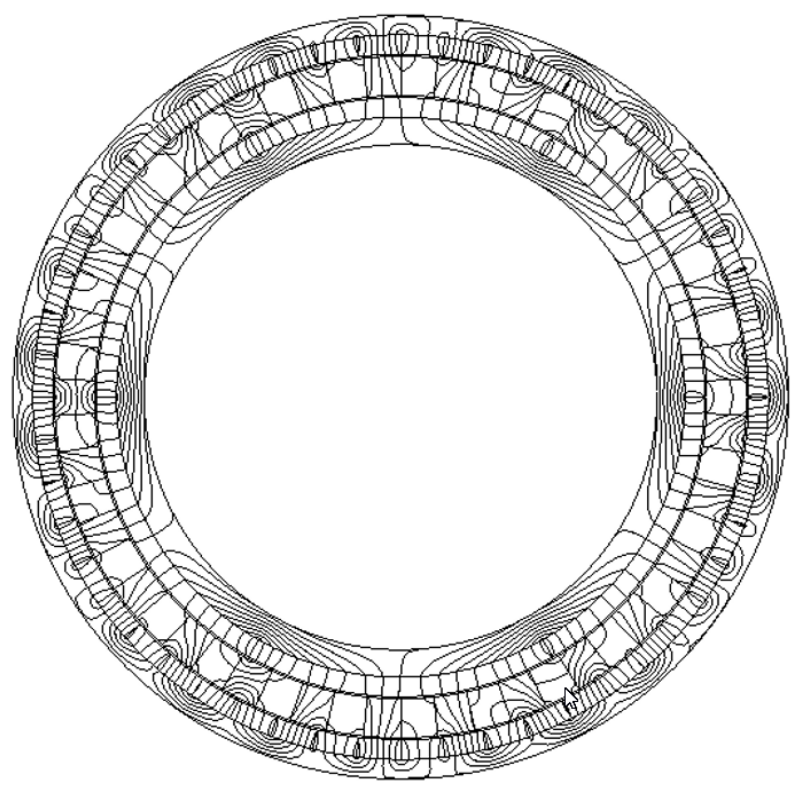

Рис. 5 - Магнитное поле редуктора для соотношения пар полюсов (3/26).

Представляется весьма технологичным выполнение модулятора как единого узла, стальные сегменты в котором соединены арками и модулятор представляет собой единый элемент, который может быть изготовлен штампованием или лазерной резкой листов электротехнической стали. В этом случае технология изготовления модулятора существенно упрощается, а, следовательно, и стоимость его изготовления [11].

Поэтому на следующем этапе исследовалось влияние размеров и расположения соединительных арок между стальными сегментами модулятора на значения максимальных моментов, действующих на высокоскоростной и низкоскоростной роторы редуктора. Размеры вставок варьировались как по толщине (1, 2 и 3 мм) так и положению (рис. 6).

Результаты моделирования приведены в табл. 2 и рис. 7. Наличие вставок между стальными сегментами сепаратора приводит к замыканию части потока в стальных сегментах и как следствие, к уменьшению передаваемого редуктором момента.

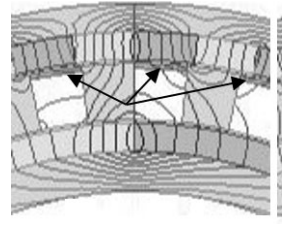

a

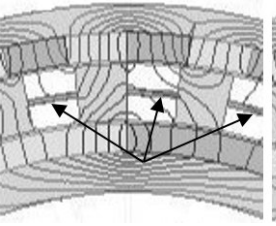

$\sigma$

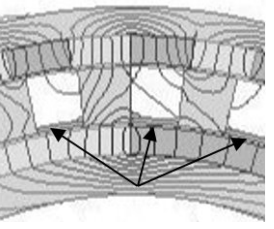

Рис. 6 - Конфигурация магнитных систем с различным расположением арок между стальными сегментами: $a$-арки в верхней части (модель MG1); $\sigma$ - арки в с средней части (модель MG2); 8 -арки в нижней части (модель MG3).

Вісник Національного технічного університету «ХПI». 


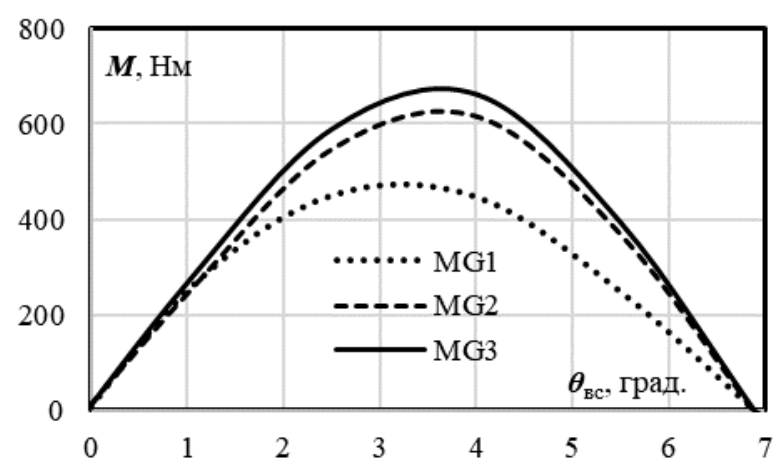

Рис. 7 - Зависимость момента на внешнем роторе редуктора от расположения вставки между стальными сегментами $\left(h_{b}=2 \mathrm{мм}\right)$.

Таблица 2- Максимальные значения момента на высокоскоростном $(\mathrm{MBc})$ и низкоскоростном $(M \mathrm{Hc})$ роторе в зависимости от толщины и расположения арки между стальными сегментами.

\begin{tabular}{|l|r|r|r|}
\hline Тип & $h_{b, \text { мм }}$ & $M$ вс, $\mathrm{H} \cdot \mathrm{M}$ & $M \mathrm{Hc}, \mathrm{H} \cdot \mathrm{M}$ \\
\hline & 0 & 85 & 723 \\
\hline \multirow{3}{*}{ MG1 } & 1 & 67 & 579 \\
\cline { 2 - 4 } & 2 & 51 & 448 \\
\cline { 2 - 4 } & 3 & 38 & 331 \\
\hline \multirow{3}{*}{ MG2 } & 1 & 76 & 657 \\
\cline { 2 - 4 } & 2 & 72 & 616 \\
\cline { 2 - 4 } & 3 & 68 & 579 \\
\hline \multirow{3}{*}{ MG3 } & 1 & 79 & 691 \\
\cline { 2 - 4 } & 2 & 76 & 660 \\
\cline { 2 - 4 } & 3 & 72 & 629 \\
\hline
\end{tabular}

Из табл. 2 следует, что наиболее оптимальной с точки зрения максимального передаваемого момента является конфигурация MG3. При этом лучший результат ожидаемо получается при наименьшей толщине арки $h_{b}=1$ мм.

При толщине арки $h_{b}=2$ мм максимальное значение электромагнитного момент на высокоскоростном роторе всего лишь на $4.5 \%$ меньше, чем при $h_{b}=$ 1 мм. Максимальное значение электромагнитного момента для «идеального» случая, когда нет соответствующих арок отличается от варианта MG3 с толщиной арок равной $h_{b}=2$ мм на $8.7 \%$. При изготовлении модулятора с точки зрения жесткости конструкции предпочтительнее вариант с толщиной арок $h_{b}=2$ мм. Следует отметить, что полученные результаты хорошо согласуется с выводами, сделанными в работе [11].

Одним из преимуществ магнитного редуктора перед механическим является то, что при перегрузке не происходит механического разрушения его элементов, просто прекращается передача механической энергии вращения от одного ротора другому. После снижения внешней нагрузки до допустимых пределов происходит восстановление вращения ведомого ротора на соответствующей скорости. На рис. 8 показан результат моделирования стабилизации скорости вращения низкоскоростного ротора магнитного редуктора с числом пар полюсов $(3 / 26)$ при работе с внешней нагрузкой 300 Нм и вращении высокоскоростного ротора с частотой вращения 8000 об/мин. Высокоскоростной и низкоскоростной роторы вращаются в противоположных направлениях. Моделирование выполнено при помощи программного пакета Infolytica Magnet.

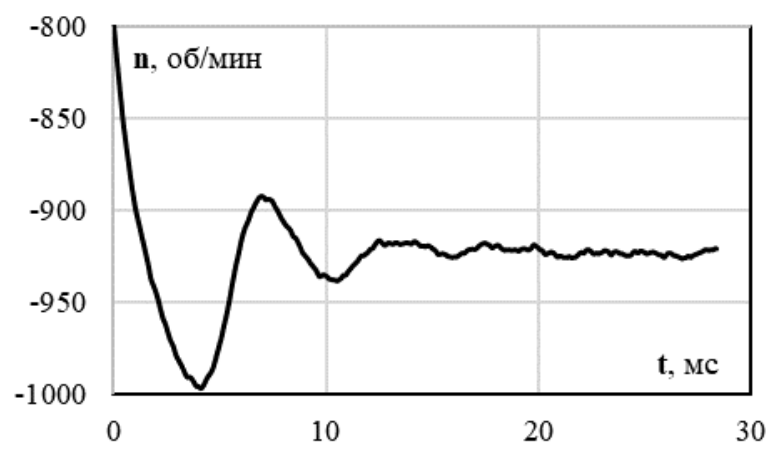

Рис. 8 - Процесс восстановления частоты вращения низкоскоростного ротора магнитного редуктора.

Таким образом, в результате численных исследований определены оптимальные геометрические параметры магнитного редуктора, при которых обеспечивается заданное значение электромагнитного момента на низкоскоростном роторе при минимальном значении зубцового момента, а также определена конфигурация модулятора, которая при существенно упрощенной технологии изготовления модулятора обеспечивает заданные характеристики редуктора. Основные технические параметры магнитного редуктора с оптимальной конфигурацией магнитной системы приведены в табл. 3.

Таблица 3- Основные технические параметры магнитного редуктора.

\begin{tabular}{|l|c|}
\hline Число пар полюсов высокоскоростного ротора & 3 \\
\hline Число пар полюсов низкоскоростного ротора & 26 \\
\hline Число неподвижных стальных сегментов & 29 \\
\hline Передаточное отношение магнитного редуктора & 8,67 \\
\hline Внешний диаметр магнитного редуктора, мм & 390 \\
\hline Внутренний диаметр магнитного редуктора, мм & 258 \\
\hline $\begin{array}{l}\text { Высота неподвижных стальных сегментов мо- } \\
\text { дулятора, мм }\end{array}$ & 20 \\
\hline Аксиальная длина редуктора, мм & 50 \\
\hline $\begin{array}{l}\text { Толщина замыкателя низкоскоростного } \\
\text { ротора, мм }\end{array}$ & 10 \\
\hline $\begin{array}{l}\text { Толщина замыкателя высокоскоростного } \\
\text { ротора, мм }\end{array}$ & 14 \\
\hline Рабочий зазоры, мм & 1 \\
\hline Тим постоянных магнитов & $\mathrm{N} 42$ \\
\hline Толщина постоянных магнитов, мм & 10 \\
\hline Максимальный момент на внешнем роторе, Н·м & 660 \\
\hline $\begin{array}{l}\text { Максимальный момент на внутреннем роторе, } \\
\text { Н·м }\end{array}$ & 76 \\
\hline $\begin{array}{l}\text { Максимальный значение зубцового момента, } \\
\text { Н·м }\end{array}$ & 2,15 \\
\hline
\end{tabular}

Вісник Наџіонального технічного університету «ХПI». 
Выводы. 1. На стадии предварительного проектирования магнитного редуктора для мотор-колеса электромобиля необходимо определять размеры магнитной системы с учетом габаритов колесного диска.

2. В силу особенностей конструкции магнитного редуктора, во время его работы возникают пульсации передаваемого крутящего момента. В результате численных исследований установлено, что при целочисленном значении коэффициента редукции зубцовый момент как минимум на порядок больше, чем для нецелочисленного значения. Так для соотношения полюсов (3/24) максимальный зубцовый момент равен $M_{3 \max }=36 \mathrm{H} \cdot$ м, для соотношения $(3 / 26)-M_{3 \max }=2,15 \mathrm{H} \cdot \mathrm{M}$, для соотношения $(3 / 27)-$ $M_{3 \max }=133 \mathrm{H} \cdot \mathrm{M}$.

3. Представляется весьма технологичным выполнение модулятора магнитного редуктора как единого узла, стальные сегменты в котором соединены арками и модулятор может быть изготовлен штампованием или лазерной резкой листов электротехнической стали. В результате численных исследований определена оптимальная толщина соединительных арок и их расположение между стальными элементами модулятора. Максимальное значение электромагнитного момента для «идеального» случая, когда нет соответствующих арок, отличается от оптимального с толщиной арок, равной $h_{b}=2$ мм, на $8,7 \%$.

\section{Список литературы}

1. Sung Jin Kim. Transfer Torque Performance Comparison in Coaxial Magnetic Gears With Different Flux-Modulator Shapes / Sung Jin Kim, Eui-Jong Park, Sang-Yong Jung, Yong-Jae Kim // IEEE Transactions on Magnetics. - 2017. - vol. 53. - No. 6. pp. 1-4. doi: 10.1109/CEFC.2016.7816184.

2. K. T. Chau. Design of a Magnetic-Geared Outer-Rotor Permanent-Magnet Brushless Motor for Electric Vehicles / K. T. Chau, D. Zhang, J. Z. Jiang, C. Liu, Y. Zhang // IEEE Transactions on Magnetics. - 2007. - vol. 43. - No. 6. - pp. - 2504-2506. doi: 10.1109/TMAG.2007.893714.

3. K. Atallah. A Novel High-Performance Magnetic Gear/K. Atallah, D. Howe. // IEEE Transactions on Magnetics. - 2001. vol. 37. - No. 4. - pp. 2844-2846.

4. W. N. Fu. A Quantitative Comparative Analysis of a Novel FluxModulated Permanent-Magnet Motor for Low-Speed Drive / W N. Fu, S. L. Ho // IEEE Transactions on Magnetics. - 2010.vol. 46. -No. 1. pp. 127-134.

doi: 10.1109/TMAG.2009.2030677.

5. C. Liu. Design Assessments of a Magnetic-Geared Double-Rotor Permanent Magnet Generator / C. Liu, H. Chung, C. Hwang // IEEE Transactions on Magnetics. - 2014. - vol. 50. - No. 1.pp. 1-4. doi: 10.1109/TMAG.2013.2279260.

6. Laxman Shah. A Variable Speed Magnetic Gear Box Using Contra-Rotating Input Shafts / Laxman Shah, Andrew Cruden, Barry W. Williams // IEEE Transactions on Magnetics. - 2013.vol. 47. - No. 2. - pp. 431-438. doi:10.1109/TMAG.2010.2097273.

7. V. Acharya. A Flux Focusing Axial Magnetic Gear / V. Acharya, J. Bird, M. Calvin // IEEE Transactions on Magnetics. - 2013. vol. 49. - No 7. - pp. 4092-4095. doi: 10.1109/TMAG.2013.2248703

8. F. T. Jorgensen. The Cycloid Permanent Magnetic Gear / F. T. Jorgensen, T. O. Andersen, P. O. Rasmussen // IEEE Trans- actions on Industry Applications. - 2008. - vol. 44. - No. 6. pp. 1659-1665. doi: 10.1109/TIA.2008.2006295.

9. X. Li. Comparison of magnetic-geared permanent-magnet machines / X. Li, K.-T. Chau, M. Cheng, W. Hua // Progress in Electromagnetics Research. - 2013. - vol. 133. - pp. 177-198.

10. S. Gerber. Cogging Torque Definitions for Magnetic Gears and Magnetically Geared Electrical Machines / S. Gerber, R. Wang // IEEE Transactions on Magnetics. - 2018. - vol. 54. - No. 4. pp. 1-9. doi: 10.1109/TMAG.2017.2784823.

11. D. Z. Abdelhamid. The Effect of Modulating Ring Design on Magnetic Gear Torque / D. Z. Abdelhamid, A. M. Knight // IEEE Transactions on Magnetics. - 2017. - vol. 53. - No. 11. - pp. 1-4. doi: 10.1109/TMAG.2017.2716920.

12. Grebenikov V.V. Design of the electric motor with permanent magnets for electric vehicle according the driving cycle / V.V. Grebenikov, M. V. Priymak // Техн. електродинаміка. 2018. - № 5. - С. 65-68.

13. Гребеников В.В. Исследование мотор-колеса для электромобиля с электродвигателем и редуктором на постоянных магнитах с учетом городского и загородного ездовых циклов / В. В. Гребеников, М.В.Прыймак // Вісник Національного технічного університету «ХПІ»: зб. наук. пр. Вип. 5 (1281) Серія: Електричні машини та електромеханічне перетворення енергії. - 2018. - С. $83-89$.

\section{References (transliterated)}

1. Sung Jin Kim, Eui-Jong Park, Sang-Yong Jung, Yong-Jae Kim. Transfer Torque Performance Comparison in Coaxial Magnetic Gears With Different Flux-Modulator Shapes // IEEE Transactions on Magnetics. 2017, vol.53, no. 6, pp.1-4. doi: 10.1109/CEFC.2016.7816184.

2. K. T. Chau, D. Zhang, J. Z. Jiang, C. Liu and Y. Zhang. Design of a Magnetic-Geared Outer-Rotor Permanent-Magnet Brushless Motor for Electric Vehicles //IEEE Transactions on Magnetics. 2007, vol. 43, no. 6, pp. 2504-2506. doi: 10.1109/TMAG.2007.893714.

3. K. Atallah, D. Howe. A Novel High-Performance Magnetic Gear // IEEE Transactions on Magnetics. 2001, vol. 37, no. 4, pp. 28442846.

4. W. N. Fu, S. L. Ho. A Quantitative Comparative Analysis of a Novel Flux-Modulated Permanent-Magnet Motor for Low-Speed Drive // IEEE Transactions on Magnetics. 2010, vol. 46, no. 1, pp. 127-134. doi: 10.1109/TMAG.2009.2030677.

5. C. Liu, H. Chung, C. Hwang. Design Assessments of a MagneticGeared Double-Rotor Permanent Magnet Generator //IEEE Transactions on Magnetics. 2014, vol. 50, no. 1, pp. 1-4. doi: 10.1109/TMAG.2013.2279260.

6. Laxman Shah, Andrew Cruden, and Barry W. Williams. A Variable Speed Magnetic Gear Box Using Contra-Rotating Input Shafts // IEEE Transactions on Magnetics. 2013, vol. 47, no. 2, pp. 431438. doi:10.1109/TMAG.2010.2097273.

7. V. Acharya, J. Bird, M. Calvin. A Flux Focusing Axial Magnetic Gear // IEEE Transactions on Magnetics. 2013, vol. 49, no 7, pp 4092-4095. doi: 10.1109/TMAG.2013.2248703.

8. F. T. Jorgensen, T. O. Andersen, P. O. Rasmussen. The Cycloid Permanent Magnetic Gear // IEEE Transactions on Industry Applications. 2008, vol. 44, no. 6, pp. 1659-1665. doi: 10.1109/TIA.2008.2006295.

9. X. Li, K.-T. Chau, M. Cheng, W. Hua. Comparison of magneticgeared permanent-magnet machines // Progress in Electromagnetics Research. 2013, vol. 133, pp. 177-198.

10. S. Gerber, R. Wang. Cogging Torque Definitions for Magnetic Gears and Magnetically Geared Electrical Machines // IEEE Transactions on Magnetics. 2018, vol. 54, no. 4, pp. 1-9. doi: 10.1109/TMAG.2017.2784823.

11. D. Z. Abdelhamid, A. M. Knight. The Effect of Modulating Ring Design on Magnetic Gear Torque // IEEE Transactions on Magnetics. 2017 , vol. 53 , no. 11 , pp. $1-4$. doi: 10.1109/TMAG.2017.2716920.

Вісник Національного технічного університету «ХПI». 
12. Grebenikov V.V., Priymak M.V. Design of the electric motor with permanent magnets for electric vehicle according the driving cycle. // Tekhn. elektrodynamika. 2018, № 5, pp. 65-68.

13. Grebenikov V.V., Priymak M.V Issledovanie motor-kolesa dlya elektromobilya s elektrodvigatelem i reduktorom na postoyannyih magnitah s uchetom gorodskogo i zagorodnego ezdovyih tsiklov [Study of a motor-wheel for an electric vehicle with an electric motor and a gear with permanent magnets, takinginto account ur- ban and countryside driving cycles] Visnyk Natsionalnoho tekhnichnoho universytetu «KhPI»: zb. nauk. pr. Vyp. 5 (1281). Seriia: Elektrychni mashyny ta elektromekhanichne peretvorennia enerhii. 2018 , pp. $83-89$.

\section{Бібліографічні описи / Библиографические описания / Bibliographic descriptions}

Дослідження впливу конфігурації магнітної системи на моментні характеристики магнітного редуктора // В. В. Гребеніков, Р. В. Гамалія // Вісник Національного технічного університету «ХПІ». Серія: "Електричні машини та електромеханічне перетворення енергії". - Х. : НТУ «ХПІ», 2019. - № 4 (1329). С. 68-74. - Бібліогр.: 13 назв. - ISSN 2409-9295.

Исследование влияния конфигурации магнитной системы на моментные характеристики магнитного редуктора // В. В. Гребеников, Р. В. Гамалея // Вісник Національного технічного університету «ХПІ». Серія: "Електричні машини та електромеханічне перетворення енергії". - Х. : НТУ «ХПІ», 2019. № 4 (1329). - С. 68-74. - Бібліогр.: 13 назв. - ISSN 2409-9295.

Investigation of the impact of the magnetic system configuration on the magnetic gear torque characteristics // V. V. Grebrnikov, R. V. Gamaliia // Bulletin of NTU "KhPI". Series: "Electric machines and electromechanical energy conversion." - Kharkiv: NTU "KhPI", 2019. - No. 4 (1329). - P. 68-74. - Bibliogr.: 13. ISSN 2409-9295.

\section{Відомості про авторів / Сведения об авторах / About the Authors}

Гребеніков Віктор Володимирович, доктор технічних наук, старший науковий співробітник, Інститут електродинаміки Національної академії наук України, провідний науковий співробітник, тел. (067)441-73-72; е-маil: elm1153@gmail.com.

Гребеніков Віктор Володимирович, доктор технических наук, старший научный сотрудник, Институт электродинамики Национальной академии наук Украины, ведущий научный сотрудник, тел. (067) 441-73-72; е-мail: elm1153@gmail.com.

Grebenikov Viktor Vladimirovich, Doctor of Technical Sciences, Senior Researcher, Institute of Electrodynamics of the National Academy of Sciences of Ukraine, Leading Researcher, tel. (067) 441-73-72; e-mail: elm1153@gmail.com.

Гамалія Ростислав Вімалійович, кандидат фізико-математичних наук, Інститут електродинаміки Національної академії наук України, старший науковий співробітник, тел: (067) 763-18-54; е-мail:rgamaleya@gmail.com.

Гамалея Ростислав Витальевич, кандидат физико-математических наук, Институт электродинамики Национальной академии наук Украины, старший научный сотрудник, тел: (067) 763-18-54; e-мail: rgamaleya@gmail.com

Gamaliia Rostyslav Vitaliyovich, Candidate of Sciences (Physics and Mathematics), Institute of Electrodynamics of the National Academy of Sciences of Ukraine, Senior Researcher, tel: (067) 763-18-54;

e-mail:rgamaleya@gmail.com 